\title{
Das Handeln der Tiere in Objekten
}

blog.kulturwissenschaften.de/das-handeln-der-tiere-in-objekten/

\section{$\underline{\text { Lisa Egeri }}$}

Mit dem Animal Turn in den Kulturwissenschaften wurde die Forderung einer Abwendung von anthropozentrischen Betrachtungsweisen formuliert und Tieren eine hohe gesellschaftliche Relevanz attestiert. Ein im Forschungsfeld der Human-Animal Studies (HAS) vertretener Ansatz zur Untersuchung der Mensch-Tier-Beziehungen besteht in der Betrachtung von Tieren als aktive Mitgestalter der geteilten Lebensräume. Wie groß ist das Handlungspotential von Tieren? Welche Formen der Agency besitzen sie? Zentral für diese Debatten ist die Akteur-Netzwerk-Theorie von Bruno Latour, für den menschliche und nicht-menschliche Akteure sowie Dinge in einem Netzwerk miteinander agieren und sich wechselseitig beeinflussen. ${ }^{1}$ In der Weiterentwicklung des Ansatzes von Latour im Hinblick auf spezifisch tierliche Aktanten, wird diesen ein Handeln auf ganz eigene Art und Weise zugesprochen, das nicht mit dem der Menschen gleichzusetzen sei. Ebenso wird das historisch gewachsene Herrschaftsverhältnis, das jede Mensch-Tier-Beziehung impliziert, berücksichtigt, um dem Tier dabei gerecht zu werden. ${ }^{2}$ Durch die Prämisse einer tierlichen Agency bekommen Tiere eine aktive Rolle bei der Ausgestaltung der Beziehungen zwischen ihnen und den Menschen sowie von sozialen und gesellschaftlichen Prozessen. Doch wie kann ihr Wirken im praktischen Kontext erfasst werden? Wie können sie sich ausdrücken, wenn sie in unserem Sinne keine Sprachfähigkeit haben und die Menschen für sie sprechen und handeln? ${ }^{3}$ Einen Zugang könnte die Untersuchung von Artefakten bieten, die Hinweise auf konkrete Interaktionen zwischen Menschen und Tieren geben.

Museen als jene Institutionen, die Objekte sammeln, bewahren, erforschen und Wissen zu ihnen vermitteln, versuchen, deren vielschichtige Sinnpotentiale zu dekodieren. Objekte erhalten somit eine Bedeutung als kulturhistorische Sachzeugen, mit denen sich in Ausstellungen Wissen vermitteln lässt. Auch in der zeitgeschichtlichen Forschung findet derzeit eine verstärkte Hinwendung zur materiellen Kultur statt, die Dingen einen Erkenntniswert zuspricht.

„Dinge werden (wieder) als Produzenten von Bedeutungen, von sozialen Beziehungen und Praktiken, von Identitäten, Wertvorstellungen und Erinnerungen betrachtet, die mit einer zunehmenden Multifunktionalität und Polysemie das Feld eindeutiger Zuordnungen verlassen haben.“4

Die Ausstellung des LWL-Industriemuseums Boten, Helfer und GefährtenBeziehungen von Mensch und Tier im Wandel befragt die zur Schau gestellten Exponate im Sinne der Human Animal Studies. Welche Rückschlüsse lassen sich auf 
die Ausgestaltung der Mensch-Tier-Beziehung ziehen? In welcher Form waren Tiere und ihre Handlungen an der Entstehung jener Artefakte beteiligt? Die Interpretation der Objekte geht damit über eine Erzählung von menschlichen Vorstellungen vom Tier oder der einseitigen Nutzung der Tiere durch den Menschen hinaus.

\section{Wilde Tiere}

Für die Beantwortung dieser Fragen bedarf es einer Differenzierung des Handlungspotenzials von Tieren in ihren Beziehungen zu Menschen je nach Funktionsraum. Ein besonders hohes Potenzial kommt dabei Wildtieren im städtischen Raum zu. Städte entstanden als Orte der menschlichen Zivilisation, in denen Tiere nur in festen, vom Menschen definierten Räumen Existenzrechte zugesprochen bekamen. Freilich gelang es nie, starre Grenzen zu ziehen, denn wildlebende Tiere fanden immer auch im städtischen Gebiet einen Lebensraum. Während heute einige freilebende Tiere als vermeintliches Anzeichen einer Rückkehr der Natur willkommen geheißen werden, gelten andere als Eindringlinge, Störenfriede oder sogar potenzielle Infektionsgefahr im Lebensraum der Menschen und werden daher verdrängt, bekämpft und vernichtet. Zu Konflikten zwischen Menschen und wildlebenden Tieren kommt es gerade dann, wenn sich Tiere eigenmächtig Räume aneignen und damit die vom Menschen gezogenen Grenzen überschreiten. Die in diesem fluiden Funktions- und Aktionsraum entstandenen Objekte erscheinen für eine Betrachtung der tierlichen Wirk- und Handlungsmacht in der Beziehung zum Menschen vielversprechend zu sein. ${ }^{5}$

\section{Nicht aufzuhalten: Wildschweine auf Nahrungssuche ${ }^{6}$}




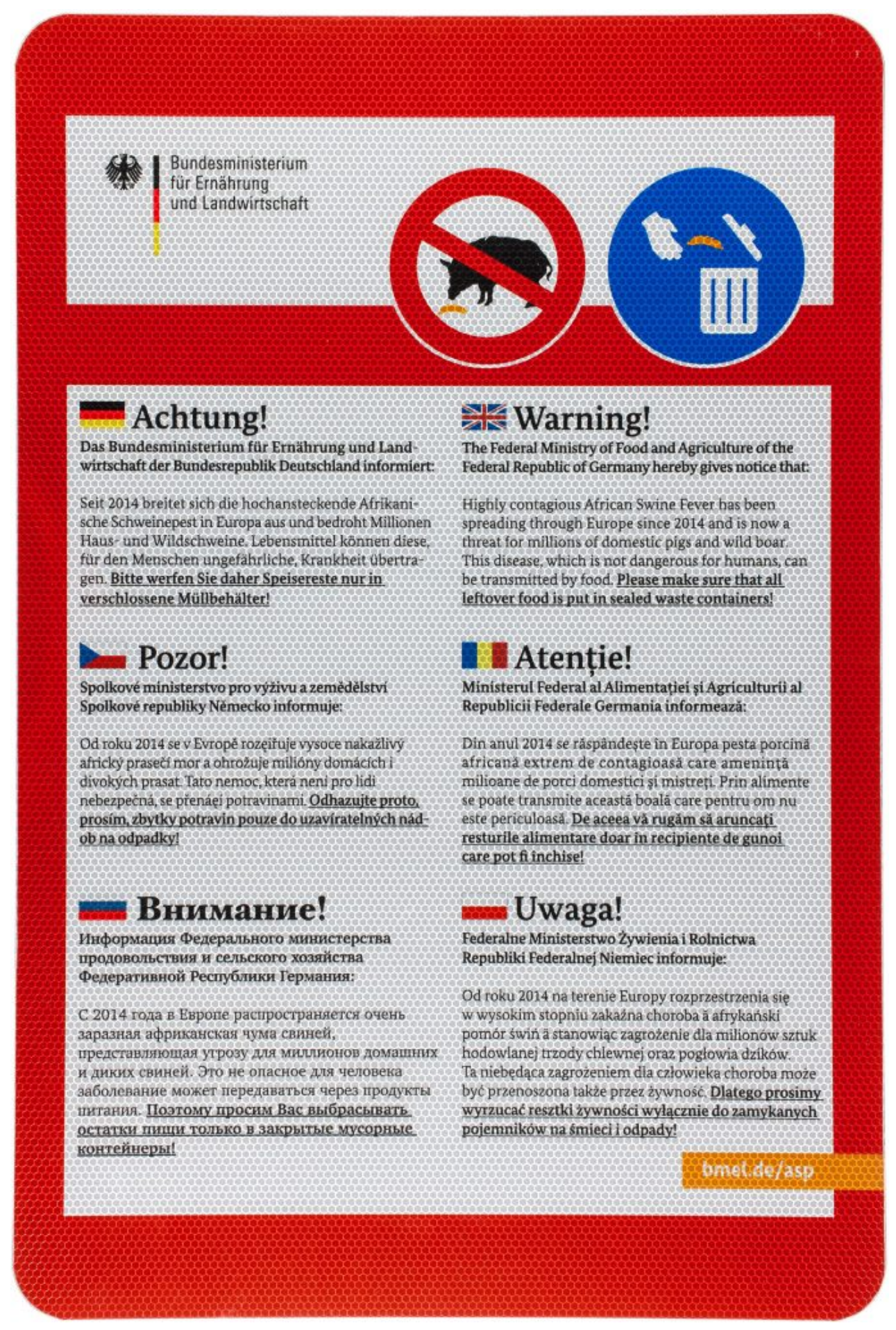

Abb.1: Hinweisschild zur Afrikanischen Schweinepest des

Bundesministeriums für Ernährung und Landwirtschaft. Foto: LWLIndustriemuseum

Wildschweine sorgen immer wieder für Aufsehen - dann ist von durchpflügten Vorgärten, zerstörten Ernten und in Panik vollzogenen Angriffen auf Menschen die Rede. Diese Akte der Zerstörung, als klare Grenzüberschreitungen der Tiere gedeutet, sorgen für ihre Wahrnehmung als Störenfriede. Immer häufiger kommt es zu diesen Konfrontationen, da den Wildschweinen immer kleinere Reviere bleiben, die oftmals durch Landstraßen und Autobahnen zerteilt werden, und sie sich immer weiter an ein Leben im Umfeld der Menschen anpassen und davon profitieren. Darüber hinaus werden sie als zu eliminierende Überträger der Afrikanischen Schweinepest als große Gefahr betrachtet. Das Schild des Bundesministeriums für Ernährung und 
Landwirtschaft klärt auf deutschen Autobahnraststätten über den Einfluss von sorglos weggeworfenem Müll über die Verbreitung der Afrikanischen Schweinepest auf. Das Virus kann auf indirektem Wege über virushaltige Essensreste übertragen werden. Wildschweine sind Allesfresser und immer auf der Suche nach Nahrungsquellen. Trotz erhöhter Jagd und der Errichtung von Zäunen gelangen sie dabei immer wieder in das Lebensumfeld der Menschen - sie sind kaum aufzuhalten. Neben Abwehrmethoden rufen die Behörden daher zur menschlichen Verhaltensänderung auf, um die Ausbreitung der vom Wildschwein übertragenen Seuche zu verhindern. Obwohl die Afrikanische Schweinepest nicht auf den Menschen übertragbar ist, schürt auch das diesjährige neue Auftreten der Seuche große Ängste. Eine Ansteckung von Hausschweinen durch Wildschweine hätte weitreichende Konsequenzen für die Nutzschweinhaltung.

\section{Überall zuhause: Tauben in der Stadt}

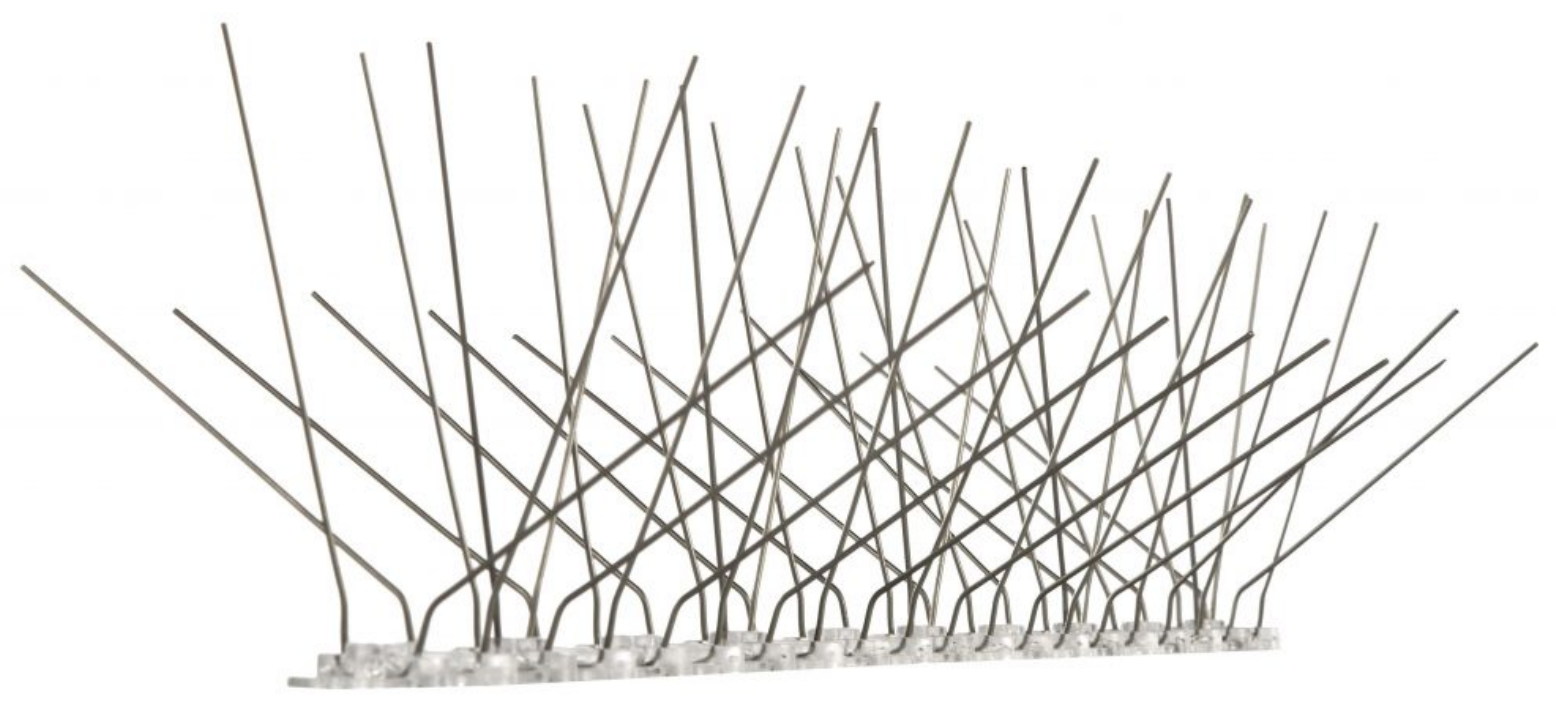

Abb.2: Spikes als Abwehrvorrichtung gegen Stadttauben. Foto: LWL-Industriemuseum

Tauben sind besonders sicht- und spürbare Wildtiere in der Stadt. Stadttauben haben sich als Kulturfolger in den Städten ,breitgemacht', wo aufgrund fehlender natürlicher Feinde, leicht zugänglicher Nahrung sowie durch ihre gute Anpassungsfähigkeit große Populationen entstanden. Überwiegend gelten sie als störend, da ihre Exkremente für hygienische und optische Probleme durch die Schädigung von Oberflächen sorgen. Mit Schutz- und Abwehrmaßnahmen gegen die Tauben wird versucht, die Trennung des Lebensraums von Tier und Mensch zu erreichen. Die Brutalität der Vergrämungsmethoden macht das Verhältnis des Menschen zum Tier besonders spürbar: Mit vielen Maßnahmen, wie den Spikes, wird ein hohes Verletzungsrisiko für die Tauben in Kauf genommen. Die Fähigkeit der Tiere, sich in allen möglichen Nischen in der Stadt einnisten zu können, prägt das Verhältnis zum Menschen und schlägt sich in diversen Abwehrobjekten nieder. 


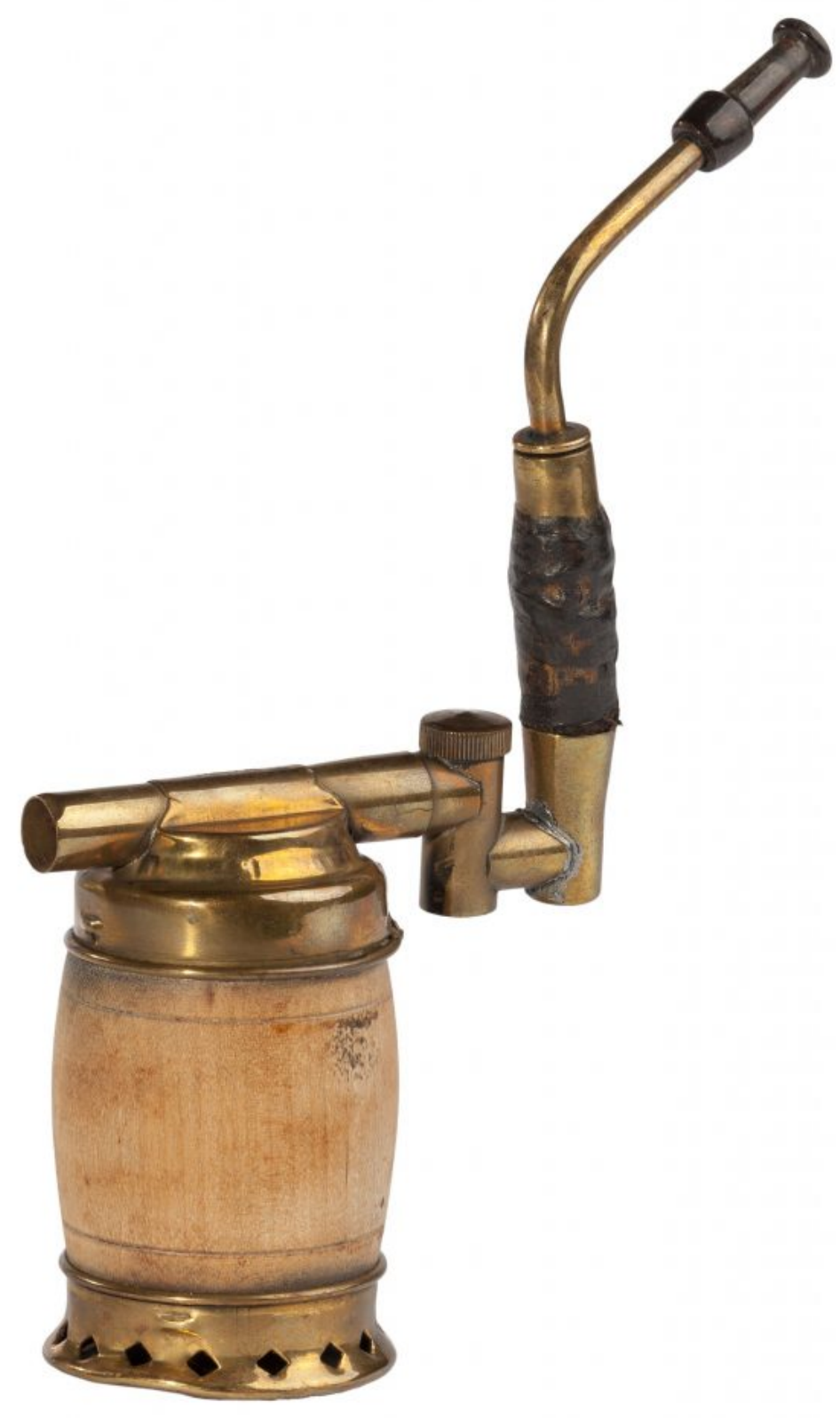

Abb.3: Imkerpfeife zur Produktion von Rauch. Foto: LWLIndustriemuseum

Trotz langjähriger Zuchtanstrengungen von Imker*innen sind Honigbienen alles andere als domestizierte Nutztiere. Die Imkerpfeife spiegelt wider, welche instinktiven Verhaltensweisen der Insekten sich der Mensch zu Nutze macht, doch insbesondere macht sie deutlich, wo die Grenzen sind und die Bienen das Verhältnis mitgestalten. Das Imkereigerät wird mit brennbarem Material gefüllt, um Rauch in den Bienenstock zu blasen. Das Bienenvolk wittert einen vermeintlichen Waldbrand und jede Biene beginnt, so viel Honig wie möglich aufzunehmen. Durch dieses Ablenkungsmanöver stören sie sich weniger an Eingriffen in ihrer Behausung. Die Notwendigkeit dieser Methode aus Sicht des Menschen erklärt sich dadurch, dass sich keine vertrauensvolle Beziehung zum Bienenvolk aufbauen lässt. Nach wie vor 
bleiben die Insekten für den Menschen unberechenbar und auch trotz Rauch können sich die Bienen immer wieder zur Wehr setzen. Imker*innen schützen sich also vor den Bienen, von denen jedes Mal eine latente Gefahr des Angriffes ausgeht, wenn der Mensch in ihren Lebensraum eindringt.

\section{Fazit}

Die Beispiele weisen darauf hin, dass die materielle Kultur - über die Materialität des Tieres hinaus - Aufschluss über die tierliche Wirk- und Handlungsmacht in konkreten Interaktionen zwischen Mensch und Tier geben kann. Die am Objekt erprobte Dekodierung der Dinge erweitert den Blick auf Mensch-Tier-Beziehungen, die in der Regel hierarchisch gestaltet sind.

Gerade museale Ausstellungen bieten die Möglichkeit, die Objekte in einen Zusammenhang zu übergeordneten Fragestellungen zu stellen und sie durch Texte, Bilder und Medien zu ergänzen, da sich die Wirkmacht des Tieres im Objekt oftmals auf den ersten Blick nicht erkennen lässt. Auch bestimmte Konstellationen von Exponaten können das Handeln der Tiere in ein neues Licht rücken. Beobachtungen von Interaktionen zwischen Mensch und Tier sowie Interviews mit menschlichen Akteuren ergänzen die Exponate durch ein komplexeres Bild von Mensch-TierBeziehungen im Rahmen konkreter Praktiken. Grade jedoch für tierhistorische Studien bietet die materielle Welt eine Vielzahl noch zu entdeckender Quellen.

\section{References}

1. Vgl. Roscher, Mieke (2016): Darf's ein bisschen mehr sein? Ein Forschungsbericht zu den historischen Human-Animal Studies. In: H-Soz-Kult, 16.12.2016, $\leq$ www.hsozkult.de/literaturereview/id/forschungsberichte-2699>. Neben der Akteur-Netzwerk-Theorie nach Latour gibt es weitere methodische Ansätze, um sich tierlicher Agency zu nähern. Detailliert setzen sich damit die Autor*innen folgenden Sammelbandes auseinander: Wirth, Sven u. a. (Hg.) (2015): Das Handeln der Tiere. Tierliche Ageny im Fokus der Human-Animal Studies, Bielefeld: transcript. https://doi.org/10.14361/9783839432266.

2. Vgl. Krebber, André/Roscher, Mieke (2016): Spuren suchen, Zeichen lesen, Fährten folgen. In: Forschungsschwerpunkt „Tier - Mensch - Gesellschaft“ (Hg.): Den Fährten folgen. Methoden interdisziplinärer Tierforschung, Bielefeld: transcript, S. 11-32. https://doi.org/10.14361/9783839433188-001.

3. Vgl. Jaeger, Friedrich (2019): Menschen und Tiere. Ein neues kulturwissenschaftliches Forschungsfeld. In: Heinrich Theodor Grütter/Ulrike Stottrop (Hg.): Mensch \& Tier im Revier, Essen: Klartext Verlag, S. 12-19.

4. Vgl. Tietmeyer, Elisabeth u.a. (Hg.) (2010): Die Sprache der Dinge. Kulturwissenschaftliche Perspektiven auf die materielle Kultur (Schriftenreihe Museum Europäischer Kulturen, Bd. 9), Münster (u.a.): Waxmann. 
5. Vgl. Steinbrecher, Aline (2016): Tiere und Geschichte. In: Roland Borgards (Hg.): Tiere. Kulturwissenschaftliches Handbuch, Stuttgart: Springer-Verlag, S. 7-15. Vgl. Brantz, Dorothee (2008): Die „animalische Stadt“: Die Mensch-TierBeziehung in der Urbanisierungsforschung. In: Informationen zur modernen Stadtgeschichte 1, S.86-100.

6. Alle folgenden Objekte werden in der Begleitpublikation zur Ausstellung eingehend beschrieben: vgl. Egeri, Lisa/Osses, Dietmar (Hg.) (2020): Boten, Helfer und Gefährten. Beziehungen von Mensch und Tier im Wandel, Essen: Klartext Verlag.

SUGGESTED CITATION: Egeri, Lisa: Das Handeln der Tiere in Objekten, in: KWIBLOG, [https://blog.kulturwissenschaften.de/das-handeln-der-tiere-in-objekten/], 26.11.2020

DOI: https://doi.org/10.37189/kwi-blog/20201126-0830 
Dieser Text wird über DuEPublico, dem Dokumenten- und Publikationsserver der Universität Duisburg-Essen, zur Verfügung gestellt. Die hier veröffentlichte Version der EPublikation kann von einer eventuell ebenfalls veröffentlichten Verlagsversion abweichen.

DOI: $\quad$ 10.37189/kwi-blog/20201126-0830

URN: urn:nbn:de:hbz:464-20201126-110918-0 